\author{
G. Burkitseterkyzy, G.L. Katkeeva, I.M. Oskembekov, \\ D.R. Gizatullina, A.M. Zhunussov \\ Zh. Abishev Chemical-Metallurgical Institute, Karaganda, Kazakhstan \\ (E-mail:katkeeva@mail.ru)
}

\title{
Study of the material composition of raw materials and the thermodynamic analysis of ore sulphidization
}

\begin{abstract}
This research is aimed at a complex solution of two problems - increasing the efficiency of processing of oxidized copper ores and utilization of sulfur-containing waste from oil and gas processing. By the methods of chemical, mineralogical and granulometric analyzes the composition of the Sayak ore and waste sulfur was examined. It was found that the total copper content in the ore made $2.7 \%, 1.8 \%$ of which is represented by oxidized minerals. The main ore minerals are malachite, azurite, brochantite and chrysocolla. A thermodynamic analysis of the interaction of oxidized copper minerals with dissolved elemental sulfur possessing the properties of nanoparticles was carried out. The possibility in principle of copper sulfidization was established. Based on the obtained data on the thermodynamic properties of polysulfide systems, $\Delta_{f} H^{0}{ }_{298.15}$, $S_{298.15}, \Delta_{f} G^{0}{ }_{298.15}, C_{p}^{0} 298.15$ of eight polysulfides $-\mathrm{CaS}_{2}, \mathrm{CaS}_{3}, \mathrm{CaS}_{4}, \mathrm{CaS}_{5}, \mathrm{CaS}_{6}, \mathrm{CaS}_{7}, \mathrm{CaS}_{8}$ и $\mathrm{CaS}_{9}$ were defined. The temperature dependences of the standard Gibbs energy of the reaction of dissolved sulfur with oxidized copper minerals - malachite, azurite, brochantite and chrysocolla - were defined. The possibility in principle of sulfidization of oxidized copper minerals with dissolved elemental sulfur possessing the properties of nanoparticles was established. It is shown that in the temperature range $298.15-500 \mathrm{~K}$ the formation of copper (II) sulfide and sulfur (IV) oxide in the interaction of oxidized copper minerals with elemental nano-sized sulfur is most likely. As the standard Gibbs energy of the sulfidation reaction increases, the minerals can be arranged in a row up to $400 \mathrm{~K}$ : azurite; malachite; brochantite; chrysocolla, after $400 \mathrm{~K}$ : azurite; brochantite; malachite; chrysocolla. In these rows, the probability of interaction with sulfur decreases.
\end{abstract}

Keywords: oxidized copper ore, sulfidization, polysulfides, sulfur-containing waste.

The problem of processing oxidized copper ores is urgent in the copper industry of Kazakhstan. It is caused by the lack of cost-effective technology for concentration of this type of raw materials. Relatively cheap flotation methods of concentration, which are traditional in the processing of sulphide raw materials, are ineffective in view of the natural hydrophilicity of oxidized copper minerals. As a result, about $20 \%$ of the total volume of mined copper mineral products that fall to the share of oxidized ores accumulate in the form of dumps and are on the balance sheet of the corresponding enterprises, requiring maintenance costs, which leads to an increase in the cost price of the final product of these enterprises [1].

The problem of sulfur utilization is caused by the excess of supply over demand in the elemental sulfur market. This is caused by the increase in the production of associated sulfur in the processing of constantly increasing volumes of sulfur-containing oil and gas raw materials and deeper purification from sulfur of the off-gases and flue gases of the coke-chemical, metallurgical and energy industries, which is dictated by the toughening of requirements for environmental protection. Forced storage of non-distributed surplus commodity sulfur, as well as storage of sulfur-containing waste in the form of dumps require large areas of territory and financial costs for maintenance by oil and gas processing enterprises. In addition, these warehouses and dumps are sources of soil and air pollution [2,3].

The solution of the problem of oxidized ores concentration is seen in the change in the properties of oxidized minerals towards the hydrophobization of the surface, which would improve the efficiency of the flotation method. One of the methods of solving the problem is chemical modification of minerals by means of sulphidization, as a result of which oxidized minerals are converted to sulphide minerals with the hydrophobic surface. For this transformation nanosized element waste sulfur can be used. In addition to the sulfiding role, nano-sulfur, depositing on the mineral surface, plays an additional hydrophobization role [4].

To determine the principle possibility of the sulfidation of oxidized copper minerals, a thermodynamic analysis of their interaction with sulfur was carried out.

\section{Chemical composition of Sayak ore}

The technological sample of Sayak deposit ore, according to mineralogical composition and genetic characteristics, also falls to the oxidized type of copper ore. The main ore minerals are malachite, azurite and chalcopyrite, the secondary ones are pyrite, magnetite, hematite, iron hydroxides. 
The chemical composition of the studied ore samples was determined by chemical and spectral analysis methods. The results are shown in Table 1.

T a b l e 1

Chemical composition of copper ore of Sayak deposit

\begin{tabular}{|c|c|c|c|}
\hline Component & Content, $\%$ & Component & Content, $\%$ \\
\hline $\mathrm{Cu}_{\text {общ. }}$ & 2.7 & $\mathrm{CaO}$ & 2.79 \\
\hline $\mathrm{Cu}_{\text {окисл. }}$ & 1.80 & $\mathrm{MgO}$ & 2.40 \\
\hline $\mathrm{SiO}_{2}$ & 56.60 & $\mathrm{~Pb}$ & 1.01 \\
\hline $\mathrm{Al}_{2} \mathrm{O}_{3}$ & 14.42 & $\mathrm{Zn}$ & - \\
\hline $\mathrm{Fe}_{\text {общ. }}$ & 6.18 & $\mathrm{~S}_{\text {общ. }}$ & 0.13 \\
\hline
\end{tabular}

In the ore, the content of oxidized copper made $66.7 \%$ of its total amount. In terms of copper, the ore is mixed.

\section{Thermodynamic analysis of the interaction of ore components with elemental sulfur}

Thermodynamic analysis of the interaction of ore components with elemental sulfur was carried out. When preparing the investigated ores for flotation concentration, their preliminary sulphidization was provided. The main sulphiding chemical is dissolved elemental sulfur, which possesses the properties of nanoparticles. The possibility in principle of interaction of this sulfur with the raw materials oxidized copper minerals is considered in the thermodynamic analysis of possible sulfidization reactions and calculation of the temperature dependence of the standard Gibbs energy of the reaction.

\section{Initial thermodynamic data}

The temperature dependences of the standard Gibbs energy of compound formation is calculated according to the equation

$$
\Delta_{f} G_{T}^{0}=\Delta_{f} H_{0}^{0}-T \Delta_{f} \Phi_{T}^{*}
$$

on the basis of the data [5-8] for the reduced Gibbs energy and the standard enthalpy of formation at $0 \mathrm{~K}$ (Tables 2, 3).

Table 2

Temperature dependence of the change in the reduced Gibbs energy of the formation of compounds

\begin{tabular}{|c|c|c|c|c|c|c|}
\hline \multirow{3}{*}{ Substance } & \multicolumn{7}{|c|}{$T, \mathrm{~K}$} \\
\cline { 2 - 7 } & 298.15 & 300 & 350 & 400 & 450 & 500 \\
\cline { 2 - 7 } & \multicolumn{7}{|c|}{$\Delta \Phi^{*}{ }_{T},{\mathrm{~J} \cdot \mathrm{mol}^{-1} \cdot \mathrm{K}^{-1}}$} \\
\hline $\mathrm{S}$ & 22.9 & 22.9 & 22.3 & 22.1 & 22.1 & 20.2 \\
\hline $\mathrm{H}_{2} \mathrm{O}$ & -164.7 & -164.7 & -164.5 & -34.5 & -37.1 & -39.7 \\
\hline $\mathrm{SO}_{2}$ & 19.5 & 19.5 & 18.0 & 16.7 & 14.9 & 13.1 \\
\hline $\mathrm{SO}_{3}$ & -63.9 & -64.0 & -66.3 & -69.6 & -71.0 & -74.9 \\
\hline $\mathrm{CO}_{2}$ & 4.1 & 4.1 & 4.0 & 3.8 & 3.7 & 3.5 \\
\hline $\mathrm{SiO}_{2}$ & -165.7 & -165.8 & -166.9 & -170.1 & -169.2 & -172.6 \\
\hline $\mathrm{Cu}_{2} \mathrm{~S}$ & 13.2 & 13.3 & 13.5 & 15.1 & 16.9 & 17.4 \\
\hline $\mathrm{CuS}$ & 20 & 2.0 & 2.0 & 1.8 & 1.1 & 0.4 \\
\hline
\end{tabular}

Ta b l e 3

Standard enthalpy of formation of compounds at 0 K

\begin{tabular}{|c|c|c|c|}
\hline Substance & $\Delta_{f} H_{0}^{0}, \mathrm{~kJ} \cdot \mathrm{mol}^{-1}$ & Substance & $\Delta_{f} H_{0}^{0}, \mathrm{~kJ} \cdot \mathrm{mol}^{-1}$ \\
\hline $\mathrm{S}$ & 13.2 & $\mathrm{CO}_{2}$ & -393.1 \\
\hline $\mathrm{H}_{2} \mathrm{O}$ & -286.3 & $\mathrm{SiO}_{2}$ & -906.0 \\
\hline $\mathrm{SO}_{2}$ & -294.3 & $\mathrm{Cu}_{2} \mathrm{~S}$ & -81.1 \\
\hline $\mathrm{SO}_{3}$ & -390.1 & $\mathrm{CuS}$ & -55.0 \\
\hline
\end{tabular}


The required values of the standard enthalpy of formation at $298.15 \mathrm{~K}[8,9]$ for calculating the temperature dependence of the standard Gibbs energy of formation by the equation are given in Table 4 .

$$
\Delta_{f} G_{T}^{0}=\Delta_{f} H_{298.15}^{0}-T \Delta_{f} S^{0}{ }_{298.15}-T M \Delta_{f} C_{p}{ }^{0}{ }_{298.15} .
$$

Ta b l e 4

\section{Standard enthalpy of formation of substances at $298.15 \mathrm{~K}$}

\begin{tabular}{|c|c|}
\hline Substance & $\Delta_{f} H_{298.15}^{0}, \mathrm{~kJ} \cdot \mathrm{mol}^{-1}$ \\
\hline $\mathrm{Cu}_{2} \mathrm{CO}_{3}(\mathrm{OH})_{2}$ & -1051.0 \\
\hline $\mathrm{Cu}_{3}\left(\mathrm{CO}_{3}\right)_{2}(\mathrm{OH})_{2}$ & -1631.3 \\
\hline $\mathrm{CuSiO}_{3} \cdot 2 \mathrm{H}_{2} \mathrm{O}$ & -1747.3 \\
\hline $\mathrm{Cu}_{4} \mathrm{SO}_{4}(\mathrm{OH})_{6}$ & -2179.9 \\
\hline
\end{tabular}

The values of the standard entropy of the formation of substances calculated using the data [8] are given in Table 5 .

The standard heat capacity of compound formation was calculated using the data [8]. Its values are given in Table 6. Data on the chrysocolla standard heat capacity are taken from [10]. The standard heat capacity of the formation of azurite, malachite and brochantite was calculated through the standard heat capacity, which was estimated using the Kumok's heat capacity increment system [11,12] by the formula

$$
C_{p 298.15}{ }^{0}\left(\mathrm{~A}_{m} \mathrm{~B}_{n}\right)=m C_{p 298,15}^{i}\left(\mathrm{~A}^{n+}\right)+n C_{p 298.15}^{i}\left(\mathrm{~B}^{m-}\right) .
$$

Table 5

Standard entropy of formation of substances

\begin{tabular}{|c|c|}
\hline Substance & $\Delta_{\mathrm{f}} \mathrm{S}_{298,15}^{0}, \mathrm{~J} \cdot \mathrm{mol}^{-1} \cdot \mathrm{K}^{-1}$ \\
\hline $\mathrm{Cu}_{2} \mathrm{CO}_{3}(\mathrm{OH})_{2}$ & -503.5 \\
\hline $\mathrm{Cu}_{3}\left(\mathrm{CO}_{3}\right)_{2}(\mathrm{OH})_{2}$ & -671.5 \\
\hline $\mathrm{CuSiO}_{3} \cdot 2 \mathrm{H}_{2} \mathrm{O}$ & -629.3 \\
\hline $\mathrm{Cu}_{4} \mathrm{SO}_{4}(\mathrm{OH})_{6}$ & -1211.0 \\
\hline
\end{tabular}

Table 6

Standard heat capacity of formation of substances

\begin{tabular}{|c|c|}
\hline Substance & $\Delta_{f} C_{p}^{0} 298.15, \mathrm{~J} \cdot \mathrm{mol}^{-1} \cdot \mathrm{K}^{-1}$ \\
\hline $\mathrm{Cu}_{2} \mathrm{CO}_{3}(\mathrm{OH})_{2}$ & -4.5 \\
\hline $\mathrm{Cu}_{3}\left(\mathrm{CO}_{3}\right)_{2}(\mathrm{OH})_{2}$ & -4.2 \\
\hline $\mathrm{CuSiO}_{3} \cdot 2 \mathrm{H}_{2} \mathrm{O}$ & 45.3 \\
\hline $\mathrm{Cu}_{4} \mathrm{SO}_{4}(\mathrm{OH})_{6}$ & -21.4 \\
\hline
\end{tabular}

On the basis of the obtained data, the temperature dependence of the standard Gibbs energy for the formation of all participants in the investigated interactions was calculated by the formulas $(1,2)$. The results are given in Table 7.

T a b l e 7

\begin{tabular}{|c|c|c|c|c|c|c|}
\hline \multirow{3}{*}{ Substance } & \multicolumn{6}{|c|}{$T, \mathrm{~K}$} \\
\hline & 298.15 & 300 & 350 & 400 & 450 & 500 \\
\hline & \multicolumn{6}{|c|}{$\Delta_{\mathrm{f}} \mathrm{G}_{\mathrm{T}}^{0}, \mathrm{~kJ} \cdot \mathrm{mol}^{-1}$} \\
\hline 1 & 2 & 3 & 4 & 5 & 6 & 7 \\
\hline $\mathrm{S}$ & 6.4 & 6.4 & 5.4 & 4.4 & 3.3 & 3.1 \\
\hline $\mathrm{H}_{2} \mathrm{O}$ & -237.2 & -236.9 & -228.7 & -225.1 & -222.1 & -219.1 \\
\hline $\mathrm{CO}_{2}$ & -394.4 & -394.4 & -394.5 & -394.7 & -394.8 & 394.9 \\
\hline $\mathrm{SiO}_{2}$ & -856.6 & -856.2 & -847.5 & -837.9 & -829.8 & -819.7 \\
\hline $\mathrm{SO}_{2}$ & -300.2 & -300.2 & -300.6 & -301.0 & -301.1 & -300.9 \\
\hline $\mathrm{SO}_{3}$ & -371.1 & -370.9 & -366.9 & -362.3 & -358.2 & -352.7 \\
\hline
\end{tabular}

Temperature dependence of the standard Gibbs energy of formation of substances 
Continuation of $\mathrm{Table} 7$

\begin{tabular}{|l|c|c|c|c|c|c|}
\hline \multicolumn{1}{|c|}{1} & 2 & 3 & 4 & 5 & 6 & 7 \\
\hline $\mathrm{Cu}_{2} \mathrm{~S}$ & -85.0 & -85.0 & -85.8 & -87.1 & -88.7 & -89.7 \\
\hline $\mathrm{CuS}$ & -55.6 & -55.6 & -55.7 & -55.7 & -55.5 & -55.2 \\
\hline $\mathrm{Cu}_{2} \mathrm{CO}_{3}(\mathrm{OH})_{2}$ & -900.9 & -900.0 & -874.8 & -849.5 & -824.3 & -799.0 \\
\hline $\mathrm{Cu}_{3}\left(\mathrm{CO}_{3}\right)_{2}(\mathrm{OH})_{2}$ & -1431.1 & -1429.9 & -1396.3 & -1362.7 & -1329.0 & -1295.4 \\
\hline $\mathrm{CuSiO}_{3} \cdot 2 \mathrm{H}_{2} \mathrm{O}$ & -1559.7 & -1558.5 & -1527.2 & -1496.3 & -1465.6 & -1435.2 \\
\hline $\mathrm{Cu}_{4} \mathrm{SO}_{4}(\mathrm{OH})_{6}$ & -1818.8 & -1816.5 & -1755.9 & -1695.1 & -1634.2 & -1573.1 \\
\hline
\end{tabular}

The temperature dependences of the standard Gibbs energy of the reaction of dissolved sulfur with oxidized copper minerals — malachite, azurite, brochantite and chrysocolla — were defined.

Thermodynamic analysis of the interaction of sulfur with brochantite

The following four interactions of dissolved sulfur with brochantite were considered:

$$
\begin{gathered}
\mathrm{Cu}_{4} \mathrm{SO}_{4}(\mathrm{OH})_{6}+4 \mathrm{~S}=2 \mathrm{Cu}_{2} \mathrm{~S}+\mathrm{SO}_{3}+2 \mathrm{SO}_{2}+3 \mathrm{H}_{2} \mathrm{O} \\
3 \mathrm{Cu}_{4} \mathrm{SO}_{4}(\mathrm{OH})_{6}+10 \mathrm{~S}=6 \mathrm{Cu}_{2} \mathrm{~S}+7 \mathrm{SO}_{3}+9 \mathrm{H}_{2} \mathrm{O} \\
\mathrm{Cu}_{4} \mathrm{SO}_{4}(\mathrm{OH})_{6}+6 \mathrm{~S}=4 \mathrm{CuS}+\mathrm{SO}_{3}+2 \mathrm{SO}_{2}+3 \mathrm{H}_{2} \mathrm{O} \\
3 \mathrm{Cu}_{4} \mathrm{SO}_{4}(\mathrm{OH})_{6}+16 \mathrm{~S}=12 \mathrm{CuS}+7 \mathrm{SO}_{3}+9 \mathrm{H}_{2} \mathrm{O}
\end{gathered}
$$

The probability of these interactions was judged by the change in the standard Gibbs energy:

$$
\begin{aligned}
& \Delta_{r} G_{T}^{0}=2 \Delta_{f} G_{T}^{0}\left(\mathrm{Cu}_{2} \mathrm{~S}\right)+\Delta_{f} G_{T}^{0}\left(\mathrm{SO}_{3}\right)+2 \Delta_{f} G_{T}^{0}\left(\mathrm{SO}_{2}\right)+3 \Delta_{f} G_{T}^{0}\left(\mathrm{H}_{2} \mathrm{O}\right)- \\
& -\Delta_{f} G_{T}^{0}\left(\mathrm{Cu}_{4} \mathrm{SO}_{4}(\mathrm{OH})_{6}\right)-4 \Delta_{f} G_{T}^{0}(\mathrm{~S}) \text {; } \\
& \Delta_{r} G_{T}^{0}=6 \Delta_{f} G_{T}^{0}\left(\mathrm{Cu}_{2} \mathrm{~S}\right)+7 \Delta_{f} G^{0}{ }_{T}\left(\mathrm{SO}_{3}\right)+9 \Delta_{f} G^{0}{ }_{T}\left(\mathrm{H}_{2} \mathrm{O}\right)- \\
& -3 \Delta_{f} G^{0}{ }_{T}\left(\mathrm{Cu}_{4} \mathrm{SO}_{4}(\mathrm{OH})_{6}\right)-10 \Delta_{f} G^{0}{ }_{T}(\mathrm{~S}) \text {; } \\
& \Delta_{r} G_{T}^{0}=4 \Delta_{f} G^{0}{ }_{T}(\mathrm{CuS})+1 \Delta_{f} G^{0}{ }_{T}\left(\mathrm{SO}_{3}\right)+2 \Delta_{f} G^{0}{ }_{T}\left(\mathrm{SO}_{2}\right)+3 \Delta_{f} G^{0}{ }_{T}\left(\mathrm{H}_{2} \mathrm{O}\right)- \\
& -\Delta_{f} G_{T}^{0}\left(\mathrm{Cu}_{4} \mathrm{SO}_{4}(\mathrm{OH})_{6}\right)-6 \Delta_{f} G_{T}^{0}(\mathrm{~S}) \\
& \Delta_{r} G_{T}^{0}=12 \Delta_{f} G^{0}{ }_{T}(\mathrm{CuS})+7 \Delta_{f} G^{0}{ }_{T}\left(\mathrm{SO}_{3}\right)+9 \Delta_{f} G^{0}{ }_{T}\left(\mathrm{H}_{2} \mathrm{O}\right)- \\
& -3 \Delta_{f} G_{T}^{0}\left(\mathrm{Cu}_{3}\left(\mathrm{Cu}_{4} \mathrm{SO}_{4}(\mathrm{OH})_{6}\right)-16 \Delta_{f} G_{T}^{0}(\mathrm{~S})\right. \text {. }
\end{aligned}
$$

\begin{tabular}{|c|c|c|c|c|c|c|c|}
\hline Reaction & $T, \mathrm{~K}$ & 298.15 & 300 & 350 & 400 & 450 & 500 \\
\hline \multirow{3}{*}{4} & $\Delta_{r} G_{\mathrm{T}}^{0}, \mathrm{~kJ} \cdot \mathrm{mol}^{-1}$ & -59.9 & -61.1 & -91.5 & -136.3 & -183.1 & -230.5 \\
\hline & Equation & \multicolumn{6}{|c|}{$\Delta_{r} G^{0}{ }_{T}=-0.843 T+195.8$} \\
\hline & $\lg K_{P}$ & 10.5 & 10.6 & 13.6 & 17.8 & 21.2 & 24.1 \\
\hline \multirow{3}{*}{5} & $\Delta_{r} G_{T}^{0}, \mathrm{~kJ} \cdot \mathrm{mol}^{-1}$ & 50.0 & 49.0 & 24.1 & -14.4 & -56.3 & -96.9 \\
\hline & Equation & \multicolumn{6}{|c|}{$\Delta_{r} G_{T}^{0}=-0.727 T+271.0$} \\
\hline & $\lg K_{P}$ & -8.8 & -8.5 & -3.6 & 1.9 & 6.5 & 10.1 \\
\hline \multirow{3}{*}{6} & $\Delta_{r} G_{\mathrm{T}}^{0}, \mathrm{~kJ} \cdot \mathrm{mol}^{-1}$ & -125.1 & -126.3 & -153.5 & -193.7 & -234.3 & -278.1 \\
\hline & Equation & \multicolumn{6}{|c|}{$\Delta_{r} G_{T}^{0}=-0.753 T+103.2$} \\
\hline & $\lg K_{P}$ & 21.9 & 22.0 & 22.9 & 25.3 & 27.2 & 29.0 \\
\hline \multirow{3}{*}{7} & $\Delta_{r} G_{\mathrm{T}}^{0}, \mathrm{~kJ} \cdot \mathrm{mol}^{-1}$ & -15.2 & -16.2 & -37.9 & -71.8 & -107.5 & -144.5 \\
\hline & Equation & \multicolumn{6}{|c|}{$\Delta_{r} G_{T}^{0}=-0.637 T+178.5$} \\
\hline & $\lg K_{P}$ & 2.7 & 2.8 & 5.6 & 9.4 & 12.5 & 15.1 \\
\hline
\end{tabular}

Substituting the values of the corresponding quantities from Table 7 to the equations, we obtained the temperature dependence of the standard Gibbs energy of the reactions (4-7). By the formula

$$
\lg K_{P(T)}=-\Delta_{r} G_{T}^{0} /(2,303 R T)
$$

the temperature dependence of the reaction equilibrium constant was calculated. The results are given in Table 8 .

T a b l e 8

Temperature dependence of the standard Gibbs energy and the equilibrium constant of the reaction of dissolved sulfur with brochantite

Based on the data in Table 8, the temperature dependences of the standard Gibbs energy of the reaction of dissolved sulfur with brochantite are shown in Figure. 


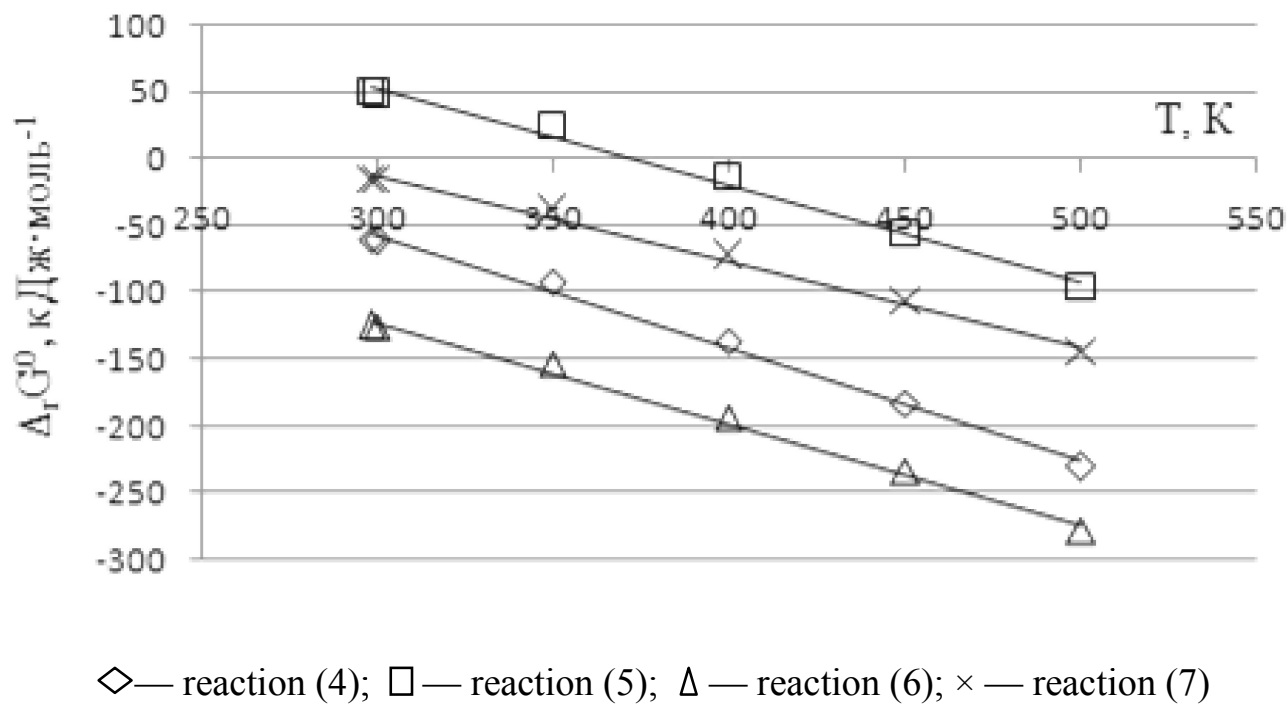

Figure. Temperature dependences of the standard Gibbs energy of the reaction of dissolved sulfur with brochantite

According to the temperature dependence of the standard Gibbs energy of the reaction, the formation of copper (II) sulphide and sulfur (IV) oxide is most likely in the interaction of brochantite with elemental nano-sized sulfur. Temperature rise increases the probability of this interaction.

The probability of reactions (4) and (7) is also high. Reaction (5) is likely with a temperature of $100{ }^{\circ} \mathrm{C}$ and higher.

\section{Conclusions}

The studies of the chemical, mineralogical and granulometric composition of the copper ore of Sayak deposit have been carried out. It has been established that the examined ore with the content of $\mathrm{Cu}_{\text {tot/ox }}=2.7 / 1.8$ is oxidized by $67 \%$.

According to the mineralogical analysis in the Sayak ore, the main ore minerals are the oxidized minerals of copper - malachite and azurite. Iron is represented in the form of minor minerals - pyrite, magnetite, hematite and iron hydroxides. According to the X-ray diffraction analysis, silicon and aluminum form nonmetallic minerals in the form of quartz, feldspar, chlorite and mica.

The results of thermodynamic analysis indicate a possibility in principle of sulfidization of oxidized copper minerals - malachite, azurite, brochantite and chrysocolla, dissolved by elemental sulfur, which possesses the properties of nanoparticles.

\section{References}

1 Смирнов С. Медные проблемы цветной металлургии / С. Смирнов // Kazakhstan. — 2010. — № 3.

2 Мировой рынок серы. [ЭР]. — Режим доступа: http://www.webeconomy.ru/index.php?newsid=1300\&page=cat\&type= news.

3 Елюбаева Ж.С. Правовые аспекты производства, хранения и транспортировки серы в Казахстане и странах СНГ / Ж.С. Елюбаева // Химическая и нефтехимическая промышленность в СНГ: ІІІ конф. — Амстердам, 2006.

4 Шайке Ж.А. Выбор оптимального режима сульфидизации окисленной медной руды / Ж.А. Шайке, Г.Л. Каткеева, И.М. Оскембеков, Д.Р. Гизатуллина, М.А. Акубаева // Промышленность Казахстана. — 2014. — № 4. — С. 68-71.

5 Гурвич Л.В. Термодинамические свойства индивидуальных веществ / Л.В. Гурвич, И.В. Вейц, В.А. Медведев и др. - М.: Наука, 1978. - Т. I, Кн. 2. - 328 с.

6 То же, 1966. - Вып. II. - 96 с.

7 Термодинамические свойства индивидуальных веществ. [ЭР]. — Режим доступа: http://www.chem.msu.su/Zn/ welcome.html.

8 Термические константы веществ. [ЭР]. - Режим доступа: http://www.chem.msu.su/cgi-bin/tkv.pl?show=welcom.html.

9 Свойства минералов. [ЭР]. - Режим доступа: http://www.74rif.ru/mineral-gl.html.

10 Бабушкин В.И. Термодинамика силикатов / В.И. Бабушкин, Г.М. Матвеев, О.П. Мчедлов-Петросян. - М.: Стройиздат, 1986. - 408 c. 
11 Кумок В.Н. Проблема согласования методов оценки термодинамических характеристик / В.Н. Кумок // Прямые и обратные задачи химической термодинамики. - Новосибирск: Наука, 1987. - С. 108-123.

12 Касенов Б.К. Термодинамические методы в химии и металлургии / Б.К. Касенов, А.С. Пашинкин, М.К. Алдабергенов. - Алматы: Рауан, 1994. — 256 с.

\title{
Г. Бүркітсетерқызы, Г.Л. Каткеева, И.М. Оскембеков, Д.Р. Гизатуллина, А.М. Жунусов Шикізаттың заттық құрамын зерттеу және кенді сульфидтеудің термодинамикалық талдауы
}

\begin{abstract}
Мақала тотыққан мыс кендерін байытудың тиімділігін арттыру және мұнай мен газ қалдықтарын өңдеу кезіндегі күкіртқұрамды қалдықтарды кәдеге жарату мәселелерін кешенді шешуге бағытталған. Химиялық, минералогиялық және гранулометриялық талдау әдістері бойынша Саяқ кенінің және қалдық күкірттің құрамдары зерттелді. Кендегі жалпы мыстың құрамы 2,7\%, оның ішінде 1,8 \% тотыққан минералдар екендігі анықталды. Негізгі кен минералдары малахит, азурит, брошантит және хризоколла болып табылады. Тотыққан мыс минералдарының, нанобөлшектік қасиеттерге ие, еріген элементтік күкіртпен әсерлесуінің термодинамикалық талдауы жүргізілді. Мыс сульфидтенуінің басты мүмкіндігі анықталды. Полисульфидтік жүйелердің термодинамикалық қасиеттері бойынша алынған нәтижелер негізінде $\mathrm{CaS}_{2}, \mathrm{CaS}_{3}, \mathrm{CaS}_{4}, \mathrm{CaS}_{5}, \mathrm{CaS}_{6}, \mathrm{CaS}_{7}, \mathrm{CaS}_{8}$ және $\mathrm{CaS}_{9}$ полисульфидтерінің $\Delta_{f} H_{298,15}^{0}, \quad S_{298,15}^{0}, \quad \Delta_{f} G_{298,15}^{0}, \quad C_{p}^{0} 298,15$ көрсеткіштері алынды. Еріген күкірттің тотыққан мыс минералдары - малахит, азурит, брошантит және хризоколламен әсерлесу реакциялары бойынша стандартты Гиббс энергиясының температуралық тәуелділіктері белгіленді. Тотыққан мыс минералдарының, нанобөлшектік қасиеттерге ие, еріген элементтік күкіртпен сульфидтенуінің негізгі мүмкіндіктері зерттелді. 298,15-500 К температура интервалында тотыққан мыс минералдарының элементтік наномөлшерлі күкіртпен әсерлесуі кезінде мыс (II) сульфиді мен күкірт (IV) оксиді түзілуінің мүмкіндігі жоғары екені анықталды. Сульфидтеу реакцияларындағы стандартты Гиббс энергияларының артуы бойынша 400 К-ге дейін мынадай қатарға орналастыруға болады: азурит,

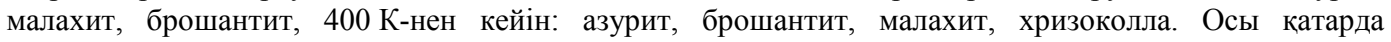
күкіртпен әрекеттесу ықтималдылығы азаяды.
\end{abstract}

Кілт сөздер: тотыққан мыс кені, сульфидтеу, полисульфидтер, күкіртқұрамды қалдықтар.

\section{Г. Буркитсетеркызы, Г.Л. Каткеева, И.М. Оскембеков, Д.Р. Гизатуллина, А.М. Жунусов}

\author{
Изучение вещественного состава сырья \\ и термодинамический анализ сульфидизации руды
}

\begin{abstract}
Статья направлена на комплексное решение двух проблем - повышение эффективности обогащения окисленных медных руд и утилизацию серосодержащих отходов переработки нефти и газа. Методами химического, минералогического, гранулометрического анализов исследованы составы саякской руды и отходной серы. Установлено, что содержание общей меди в руде 2,7 \%, из них 1,8 \% представлено окисленными минералами. Главные рудные минералы представлены малахитом, азуритом, брошантитом и хризоколлой. Проведен термодинамический анализ взаимодействия окисленных медных минералов с растворенной элементной серой, обладающей свойствами наночастиц. Установлена принципиальная возможность сульфидизации меди. На основе полученных данных по термодинамическим свойствам полисульфидных систем определены $\Delta_{f} H_{298,15}^{0}, S_{298,15}^{0}, \Delta_{f} G_{298,15}^{0}, C_{p}^{0} 298,15$ восьми полисульфидов - $\mathrm{CaS}_{2}, \mathrm{CaS}_{3}, \mathrm{CaS}_{4}, \mathrm{CaS}_{5}, \mathrm{CaS}_{6}, \mathrm{CaS}_{7}, \mathrm{CaS}_{8}$ и $\mathrm{CaS}_{9}$. Кроме того, установлены температурные зависимости стандартной энергии Гиббса реакции растворенной серы с окисленными медными минералами - малахитом, азуритом, брошантитом и хризоколлой. Указана принципиальная возможность сульфидизации окисленных медных минералов растворенной элементной серой, обладающей свойствами наночастиц. Показано, что в температурном интервале 298,15-500 К при взаимодействии окисленных медных минералов с элементной наноразмерной серой наиболее вероятно образование сульфида меди (II) и оксида серы (IV). По мере возрастания стандартной энергии Гиббса реакции сульфидирования минералы можно расположить в ряд до 400 К: азурит, малахит, брошантит, хризоколла, после $400 \mathrm{~K}$ : азурит, брошантит, малахит, хризоколла. В данных рядах уменьшается вероятность взаимодействия с серой.
\end{abstract}

Ключевые слова: окисленная медная руда, сульфидизация, полисульфиды, серосодержащие отходы. 


\section{References}

1 Smirnov, S. (2010). Mednye problemy tsvetnoi metallurhii [Copper Problems of Non-Ferrous Metallurgy]. Kazakhstan, 3 [in Russian].

2 Mirovoi rynok sery [Global sulfur market] (n.d.). www.webeconomy.ru. Retrieved from http://www.webeconomy.ru /index.php?newsid= 1300\&page=cat\&type=news [in Russian].

3 Yelyubayeva, Zh.S. (2006). Pravovye aspekty proizvodstva, khraneniia i transportirovki sery v Kazakhstane i stranakh SNG [Legal aspects of sulfur production, storage and transportation in Kazakhstan and CIS countries]. Proceedings from Chemical and petrochemical industry in the CIS '06: III konferentsiia - IIIrd Conference. Amsterdam [in Russian].

4 Shaike, Zh.A., Katkeyeva, G.L., Oskembekov I.M., Gizatullina, D.R., \& Akubayeva, M.A. (2014). Vybor optimalnoho rezhima sulfidizatsii okislennoi mednoi rudy [Choice of optimal mode of sulfidization of oxidized copper ore]. Promyshlennost Kazakhstana - Industry of Kazakhstan, 4, 68-71 [in Russian].

5 Gurvich, L.V., Veits, I.V., Medvedev, V.A. et al. (1978). Termodinamicheskie svoistva individualnykh veshchestv [Thermodynamic properties of individual substances]. (Iss. I, Book 2). Moscow: Nauka [in Russian].

6 As above (1966). (Iss. II) [in Russian].

7 Termodinamicheskie svoistva individualnykh veshchestv [Thermodynamic properties of individual substances] (n.d.). www.chem.msu.su Retrieved from http://www.chem.msu.su/ Zn/welcome.html [in Russian].

8 Termicheskie konstanty veshchestv [Thermal constants of substances] (n.d.). www.chem.msu.su Retrieved from http://www.chem.msu.su/cgi-bin/tkv.pl? show=welcom.html [in Russian].

9 Svoistva mineralov [Properties of minerals] (n.d.). www.74rif.ru Retrieved from http://www.74rif.ru/mineral-gl.html [in Russian].

10 Babushkin, V.I., Matveyev, G.M., \& Mchedlov-Petrosyan, O.P. (1986). Termodinamika silikatov [Thermodynamics of silicates]. Moscow: Stroiizdat [in Russian].

11 Kumok, V.N. (1987). Problema sohlasovaniia metodov otsenki termodinamicheskikh kharakteristik [The problem of coordination of methods for estimating thermodynamic characteristics]. Priamye i obratnye zadachi khimicheskoi termodinamiki - Direct and inverse problems of chemical thermodynamics. Novosibirsk: Nauka [in Russian].

12 Kassenov, B.K., Pashinkin, A.S., \& Aldabergenov, M.K. (1994). Termodinamicheskie metody v khimii $i$ metallurgii [Thermodynamic methods in chemistry and metallurgy]. Almaty: Rauan [in Russian]. 